\title{
Papilopatia diabética e triamcinolona intravítrea
}

\section{Diabetic papillopathy and intravitreal triamcinolone}

Eduardo de França Damasceno', Nadyr Antonia Pereira Damasceno², Soraya Alessandra Horowitz ${ }^{3}$,Octavio Moura Brasil do Amaral Filho ${ }^{4}$

\section{RESUMO}

Os autores descrevem um caso de papilopatia diabética em paciente diabética insulinodependente no qual se utilizou o acetonido de triancinolona com injeção intravítrea como opção de tratamento.

Descritores: Acetonido de triancinolona/uso terapêutico; Papiledema/quimioterapia; Papiledema/etiologia; Diabetes mellitus/complicações,Acuidade visual; Relatos de casos [Tipo de publicação]

\footnotetext{
'Médico do Setor de Retina e Vítreo do Hospital Universitário Clementino Fraga Filho da Universidade Federal do Rio de Janeiro - UFRJ - Rio de Janeiro (RJ), Brasil;

${ }^{2}$ Médico do setor de Retina e Vítreo do Hospital Naval Marcílio Dias - Rio de Janeiro (RJ), Brasil;

${ }^{3}$ Médico do setor de Retina e Vítreo do Hospital Naval Marcílio Dias - Rio de Janeiro (RJ), Brasil;

${ }^{4}$ Professor Adjunto do Hospital Universitário Clementino Fraga Filho da Universidade Federal do Rio de Janeiro - UFRJ - Rio de Janeiro (RJ), Brasil.
} 


\section{INTRODUÇÃO}

A papilopatia diabética, inicialmente descrita por Lubow ${ }^{(1)}$,é definida como edema de disco óptico que ocorre em pacientes diabéticos do tipo I ou II de forma usualmente transitória, unilateral ou bilateral associada, na maior parte das vezes, com mínimo ou nenhum deficit visual ${ }^{(2)}$. Há relatos de casos associados ou não com retinopatia diabética ${ }^{(3)}$, bem como casos similares descritos como mimetizando edema macular clinicamente significativo devido à sua extensão sobre a região papilomacular ${ }^{(4)}$. Devido à característica fugaz não é comumente recomendado nenhum tipo de tratamento a não ser um adequado controle glicêmico ${ }^{(3)}$. Este relato expõe uma proposta alternativa para o manejo da papilopatia diabética com o uso intraocular de acetonido de triancinolona em casos de diminuição da acuidade visual.

\section{Relato de caso}

Mulher de 45 anos, diabética (tipo I) insulinodependente, há 12 anos, com controle metabólico irregular, apresentando queixas de halos visuais há 1 semana após descontrole glicêmico $(330 \mathrm{mg} / \mathrm{dl})$.

Ao exame oftalmológico apresentava acuidade visual com correção de 20/40 no olho direito (OD) e 20/ 20 no olho esquerdo (OE); reflexo pupilar fotomotor normal; biomicroscopia e pressão intra-ocular (PIO) normais em ambos os olhos (AO). Com ultrassonografia $\mathrm{B}^{5}$ de olho direito apresentando elevação do disco óptico, sem sinal de aumento da escavação ou presença de calcificação; com olho esquerdo normal. Ao exame de fundoscopia apresentava edema e hiperemia de disco óptico no OD (Figuras 1 e 2).

Apresentava-se com exame de campimetria computadorizada inexpressivo, com discreto aumento de mancha cega no OD. Exame de tomografia computadorizada (TC) de crânio e parecer neurológico com ausência de alterações. Não havia sinais de retinopatia diabética ao exame fundoscópico ou angiográfico.Ao exame retinográfico com contraste fluorescente revelou extravasamento de contraste no disco óptico direito. Conforme não havia sinal de alteração de reflexo fotomotor direto e consensual neste mesmo olho, foi aventada a hipótese diagnóstica de Papilopatia Diabética. Foi feito um controle glicêmico junto com endocrinologista, durante o período de acompanhamento.

Foi realizado procedimento de injeção intravítrea de triancinolona (Kenalog $-40 \mathrm{mg} / \mathrm{ml}$ ) - 0,1 ml intravítrea no OD, com mais uma injeção consecutiva com intervalo de 2 meses de acompanhamento.

A evolução do quadro da papilopatia diabética

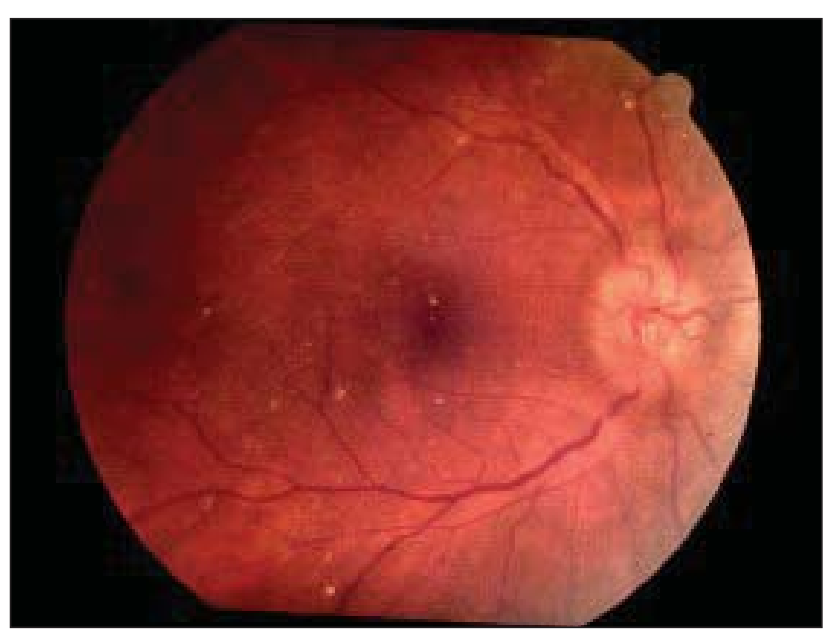

Figura 1: Papilopatia - Disco óptico OD

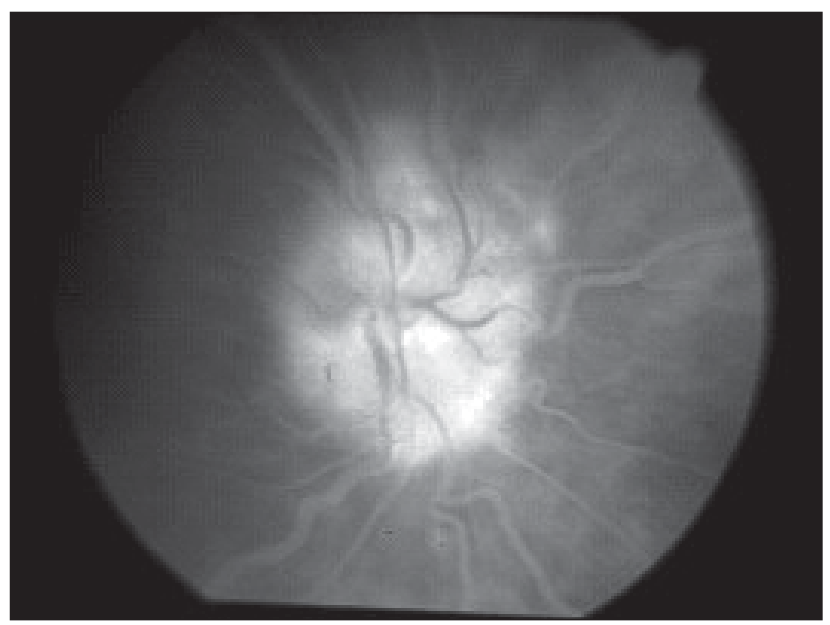

Figura 2: Angiografia - Disco Óptico OD

se sucedeu com acuidade visual melhorando para 20/25 no OD após um período de 8 meses do quadro inicial, com campo visual novamente inexpressivo (dentro da normalidade) e resolução do quadro de edema papilar e hiperemia.

\section{Discussão}

Uma síndrome de edema de disco óptico de caráter transitório e idiopático, com mínima ou sem evidência clínica de permanente baixa visual, afetando usualmente diabéticos jovens, foi primeiramente descrita em 1971, por Lubow and Makley, e na literatura nacional por Souza Filho et al., em $1981^{(1,6)}$. A incidência desta entidade é difícil de ser estimada devido à sua baixa constância, havendo relatos de uma freqüência de $0,4 \%$ entre todos os pacientes diabéticos, observados em trabalhos de pequena casuística ${ }^{(2.3)}$. Disto denota pela raridade, a 
dificuldade de grandes estudos para determinação de sua etiopatogenia e terapêutica.

O diagnóstico é de exclusão clínica, visto haver outras entidades causadoras de edema de disco óptico em pacientes diabéticos, como hipertensão intracraniana, hipertensão arterial, e neuropatia óptica isquêmica anterior não arterítica (NOIA) ou mesmo a forma arterítica ${ }^{(7)}$. Tais patologias foram descartadas através de tomografia computadorizada de crânio, reflexo pupilar fotomotor, campo visual computadorizado, exame de sangue e exame clínico geral. Havendo concordância com estes critérios neste relato de caso.

A diferenciação com pseudopapiledema causado por drusas de papila foi realizada através da fotografia anerita, que evidenciou ausência de lesões autofluorescentes, campo visual computadorizado sem alterações compatíveis e ultrassonografia B com ausência de imagem compatível com drusas.

Como são pequenos os números de publicações pertinentes à papilopatia diabética, não há uma regra clássica de procedimentos terapêuticos, sendo a própria involução espontânea sem total garantia de prognóstico favorável em todos os casos, podendo ocorrer baixa da acuidade visual no decorrer ou após o quadro da papilopatia diabética.A paciente em tela evoluiu para acuidade de 20/25 em olho direito, mantendo 20/20 em olho esquerdo.

O uso de corticóides sistêmicos ou tópicos na papilopatia diabética foi pouco comentado até 2004, quando foi descrito o uso da triancinolona intravítrea como tratamento para os casos em que a exuberância do extravasamento capilar peripapilar acomete a região macular, ocasionando edema de mácula associado ou não à retinopatia diabética, sendo recomendado nos casos de amplo acometimento de acuidade visual ${ }^{(4)}$.

Conforme relatos atuais, a papilopatia diabética pode atuar como fator prognóstico ${ }^{(8)}$, e embora existam relatos substanciais de bom prognóstico em relação a preservação da acuidade e campo visual, acredita-se que a triancinolona possa atuar como mediador de limitação de tempo de evolução desta entidade, pelas suas propriedades anti-inflamatória e estabilizadora de perfusão capilar ${ }^{(0.10)}$.

No caso relatado, apesar de haver uma melhora anatômico-visual após um período relativamente longo de 8 meses, houve mais precocemente uma melhora da acuidade visual logo após 30 dias da segunda aplicação da triancinolona intravítrea.

Desta forma, acredita-se na validade deste tipo de procedimento, bem como se sucedeu uma evolução satisfatória da Papilopatia Diabética no caso descrito. Do mesmo modo, uma vez que há controvérsias sobre a real etiologia desta entidade, até em relação a outra entidade afim, como a neuropatia óptica isquêmica ${ }^{(11)}$, acredita-se que haja necessidade de maior pesquisa para fundamentar esta opção terapêutica com triancinolona. Disto, se resume que esta entidade ainda com padrões semiológicos e terapêuticos,pouco definidos, ainda requer maior pesquisa para definições mais abalizadas. Entretanto,concordase de que prós e contras da terapia devem ser analisados e expostos ao paciente, caso a caso.

\section{Abstract}

The authors describe one case of diabetic papillopathy. They used an intravitreal triamcinolone as an option of treatment.

Keywords: Triancinolone acetonide/therapeutic use; Papilledema/drug therapy; Papilledema/etiology; Diabetes mellitus/complications; Visual acuity; Case reports [Publication type]

\section{RefERENCIAS}

1. Lubow M, Makley TA Jr. Pseudopapilledema of juvenile diabetes mellitus. Arch Ophthalmol. 1971; 85(4): 417-22.

2. Barr CC, Glaser JS, Blankenship G. Acute disc swelling in juvenile diabetes. Clinical profile and natural history of 12 cases. Arch Ophthalmol. 1980; 98(12): 2185-92.

3. Regillo CD, Brown GC, Savino PJ, Byrnes GA, Benson WE, Tasman WS, Sergott RC. Diabetic papillopathy. Patient characteristics and fundus findings. Arch Ophthalmol. 1995; 113(7): 889-95.

4. Al-Haddad CE, Jurdi FA, Bashshur ZF. Intravitreal triamcinolone acetonide for the management of diabetic papillopathy. Am J Ophthalmol. 2004; 137(6): 1151-3.

5. Byrne SF, Green RL. Ultrasound of the eye and orbit. 2nd ed. St. Louis: Mosby; 2002. p. 434-7.

6. Souza Filho JL, Bonomo PP, Imamura PM. Papilopatia diabética (estudo clinico e angiográfico de sua involução). Arq Bras Oftalmol. 1981; 44(3):113-6,.

7. Vaphiades MS. The disk edema dilemma. Surv Ophthalmol. 2002; 47(2) :183-8. Comment in: Surv Ophthalmol. 2002; 47(6):600-2; author reply 602

8. Bayraktar Z, Alacali N, Bayraktar S. Diabetic papillopathy in type II diabetic patients. Retina. 2002; 22(6):752-8. Comment in: Retina. 2004; 24(1):183-4; author reply 184.

9. Massin P, Audren F, Haouchine B, Erginay A, Bergmann JF, Benosman R, et al. Intravitreal triamcinolone acetonide for diabetic diffuse macular edema: preliminary results of a prospective controlled trial. Ophthalmology. 2004; 111(2):21824; discussion 224-5.

10. Martidis A, Duker JS, Greenberg PB, Rogers AH, Puliafito $\mathrm{CA}$, Reichel E, Baumal C. Intravitreal triamcinolone for refractory diabetic macular edema. Ophthalmology. 2002; 109(5):920-7.

11. Hayreh SS. Diabetic papillopathy and nonarteritic anterior ischemic optic neuropathy. Surv Ophthalmol. 2002; 47(6): $600-2$; author reply 602.

\author{
ENDEREÇO PARA CORRESOPONDÊNCIA: \\ Eduardo F. Damasceno \\ Rua Jangadeiros, 40 - Apto. 601 \\ CEP 22420-010 - Rio de Janeiro - RJ \\ e-mail: e_damasceno@yahoo.com
}

